\title{
RETRACTED ARTICLE: Color image watermarking in big multimedia data applications
}

\author{
Ju Zhu ${ }^{1}$ - Chao Xiong ${ }^{1,2} \cdot$ Hongwei $\mathrm{Du}^{2} \cdot$ Ruxi Xiang ${ }^{2} \cdot$ \\ Yuan $\mathbf{L i}^{2}$
}

Received: 14 December 2017 / Revised: 27 December 2017 / Accepted: 29 December 2017 / Published online: 7 February 2018

(C) Springer Science+Business Media, LLC, part of Springer Nature 2018

This article is retracted at the request of Editor-in-Chief because its content has been duplicated from unpublished manuscripts authored by Pegah Nikbakht Bideh, Mojtaba Mahdavi, Shahrm Etemadi, and Sima Arasteh without permission. Corresponding Author Chao Xiong agrees to this retraction. Authors Ju Zhu, Hongwei Du, Ruxi Xiang, and Yuan Li have not responded to any correspondence from the editor or publisher about this retraction.

The online version of this article contains the full text of the retracted article as electronic supplementary material.

Electronic supplementary material The online version of this article (https://doi.org/10.1007/s11042-0175604-y) contains supplementary material, which is available to authorized users.

\section{Chao Xiong}

xiongchao1778@126.com

1 The Key Laboratory of Electronic Measurement Technology, The 41st Institute of CETC, BengBu, Anhui Province 233010, People's Republic of China

2 School of Electrical and Photoelectronic Engineering, Changzhou Institute of Technology, Changzhou, Jiangsu Province 213032, China 